\title{
ALGEBRAIC CYCLES AND FANO THREEFOLDS OF GENUS 8
}

\author{
ROBERT LATERVEER
}

\begin{abstract}
We show that prime Fano threefolds $Y$ of genus 8 have a multiplicative ChowKünneth decomposition, in the sense of Shen-Vial. As a consequence, a certain tautological subring of the Chow ring of powers of $Y$ injects into cohomology.
\end{abstract}

\section{INTRODUCTION}

Given a smooth projective variety $Y$ over $\mathbb{C}$, let $A^{i}(Y):=C H^{i}(Y)_{\mathbb{Q}}$ denote the Chow groups of $Y$ (i.e. the groups of codimension $i$ algebraic cycles on $Y$ with $\mathbb{Q}$-coefficients, modulo rational equivalence). The intersection product defines a ring structure on $A^{*}(Y)=\bigoplus_{i} A^{i}(Y)$, the Chow ring of $Y$. Motivated by the cases of K3 surfaces and abelian varieties, Beauville [1] has conjectured that for certain special varieties, the Chow ring should admit a multiplicative splitting. To make concrete sense of Beauville's elusive "splitting property conjecture", ShenVial [40] have introduced the concept of multiplicative Chow-Künneth decomposition. It seems both interesting and difficult to better understand the class of special varieties admitting such a decomposition (for some partial answers, cf. subsection 2.1 below).

This paper aims to contribute to this program. The main result is as follows:

Theorem (=Theorem 3.1). Let $Y$ be a Fano threefold that is prime (i.e., the Picard group is generated by the canonical divisor) of genus 8 . Then $Y$ has a multiplicative Chow-Künneth decomposition.

The argument proving Theorem 3.1 relies on the classical fact that $Y$ is birational to a cubic threefold (cf. subsection 2.3 below), plus the recently established fact that cubic threefolds have a multiplicative Chow-Künneth decomposition [7], [11].

We check that the multiplicative Chow-Künneth decomposition we construct behaves well with respect to the birationality with the cubic threefold (Proposition 4.1), and also with respect to birationalities among two genus 8 Fano threefolds (Proposition 4.2).

Using Theorem 3.1, we obtain a result concerning the tautological ring, which is a certain subring of the Chow ring of powers of $Y$ :

Corollary (=Corollary 5.1). Let $Y$ be a prime Fano threefold of genus 8 , and $m \in \mathbb{N}$. Let

$$
R^{*}\left(Y^{m}\right):=\left\langle\left(p_{i}\right)^{*}(h),\left(p_{i j}\right)^{*}\left(\Delta_{Y}\right)\right\rangle \subset A^{*}\left(Y^{m}\right)
$$

Key words and phrases. Algebraic cycles, Chow group, motive, Bloch-Beilinson filtration, Beauville's "splitting property" conjecture, multiplicative Chow-Künneth decomposition, Fano threefolds, tautological ring.

2020 Mathematics Subject Classification: 14C15, 14C25, 14C30.

Supported by ANR grant ANR-20-CE40-0023. 
be the $\mathbb{Q}$-subalgebra generated by pullbacks of the polarization $h \in A^{1}(Y)$ and pullbacks of the diagonal $\Delta_{Y} \in A^{3}(Y \times Y)$. The cycle class map induces injections

$$
R^{*}\left(Y^{m}\right) \hookrightarrow H^{*}\left(Y^{m}, \mathbb{Q}\right) \text { for all } m \in \mathbb{N} .
$$

Corollary 5.1 can be loosely paraphrased by saying that prime genus 8 Fano threefolds behave like hyperelliptic curves from the point of view of intersection theory (cf. Remark 5.2 below).

To close this introduction, here is an open question:

Question 1.1. Let $Y$ be a Fano threefold with $\operatorname{Pic}(Y)=\mathbb{Z}$. Does $Y$ have a multiplicative Chow-Künneth decomposition? (And is Corollary 5.1 true for $Y$ ?)

(Without the hypothesis on $\operatorname{Pic}(Y)$, the answer can be negative, as suggested by [1, Example 1.7].) In the final section 6 , we record another question on a related note.

Conventions. In this article, the word variety will refer to a reduced irreducible scheme of finite type over $\mathbb{C}$. A subvariety is a (possibly reducible) reduced subscheme which is equidimensional.

All Chow groups will be with rational coefficients: we will denote by $A_{j}(Y)$ the Chow group of $j$-dimensional cycles on $Y$ with $\mathbb{Q}$-coefficients; for $Y$ smooth of dimension $n$ the notations $A_{j}(Y)$ and $A^{n-j}(Y)$ are used interchangeably. The notation $A_{\text {hom }}^{j}(Y)$ will be used to indicate the subgroup of homologically trivial cycles. For a morphism $f: X \rightarrow Y$, we will write $\Gamma_{f} \in$ $A_{*}(X \times Y)$ for the graph of $f$.

The contravariant category of Chow motives (i.e., pure motives with respect to rational equivalence as in [39], [34]) will be denoted $\mathcal{M}_{\text {rat }}$.

\section{Preliminaries}

\subsection{MCK decomposition.}

Definition 2.1 (Murre [33]). Let $X$ be a smooth projective variety of dimension $n$. We say that $X$ has a $\mathrm{CK}$ decomposition if there exists a decomposition of the diagonal

$$
\Delta_{X}=\pi_{X}^{0}+\pi_{X}^{1}+\cdots+\pi_{X}^{2 n} \text { in } A^{n}(X \times X),
$$

such that the $\pi_{X}^{i}$ are mutually orthogonal idempotents and $\left(\pi_{X}^{i}\right)_{*} H^{*}(X, \mathbb{Q})=H^{i}(X, \mathbb{Q})$.

(NB: "CK decomposition" is shorthand for "Chow-Künneth decomposition".)

Remark 2.2. The existence of a CK decomposition for any smooth projective variety is part of Murre's conjectures [33], [19].

Definition 2.3 (Shen-Vial [40]). Let $X$ be a smooth projective variety of dimension $n$. Let $\Delta_{X}^{s m} \in A^{2 n}(X \times X \times X)$ be the class of the small diagonal

$$
\Delta_{X}^{s m}:=\{(x, x, x) \mid x \in X\} \subset X \times X \times X .
$$

An MCK decomposition is a CK decomposition $\left\{\pi_{X}^{i}\right\}$ of $X$ that is multiplicative, i.e. it satisfies

$$
\pi_{X}^{k} \circ \Delta_{X}^{s m} \circ\left(\pi_{X}^{i} \times \pi_{X}^{j}\right)=0 \text { in } A^{2 n}(X \times X \times X) \text { for all } i+j \neq k .
$$

(NB: "MCK decomposition" is shorthand for "multiplicative Chow-Künneth decomposition".) 
Remark 2.4. The small diagonal (seen as a correspondence from $X \times X$ to $X$ ) induces the multiplication morphism

$$
\Delta_{X}^{s m}: \quad h(X) \otimes h(X) \rightarrow h(X) \text { in } \mathcal{M}_{\text {rat }} .
$$

Suppose $X$ has a CK decomposition

$$
h(X)=\bigoplus_{i=0}^{2 n} h^{i}(X) \text { in } \mathcal{M}_{\text {rat }}
$$

By definition, this decomposition is multiplicative if for any $i, j$ the composition

$$
h^{i}(X) \otimes h^{j}(X) \rightarrow h(X) \otimes h(X) \stackrel{\Delta_{X}^{s m}}{\longrightarrow} h(X) \text { in } \mathcal{M}_{\text {rat }}
$$

factors through $h^{i+j}(X)$.

If $X$ has an MCK decomposition, then setting

$$
A_{(j)}^{i}(X):=\left(\pi_{X}^{2 i-j}\right)_{*} A^{i}(X),
$$

one obtains a bigraded ring structure on the Chow ring: that is, the intersection product sends $A_{(j)}^{i}(X) \otimes A_{\left(j^{\prime}\right)}^{i^{\prime}}(X)$ to $A_{\left(j+j^{\prime}\right)}^{i+i^{\prime}}(X)$.

It is expected that for any $X$ with an MCK decomposition, one has

$$
A_{(j)}^{i}(X) \stackrel{? ?}{=} 0 \text { for } j<0, \quad A_{(0)}^{i}(X) \cap A_{h o m}^{i}(X) \stackrel{? ?}{=} 0 ;
$$

this is related to Murre's conjectures $B$ and $D$, that have been formulated for any CK decomposition [33].

The property of having an MCK decomposition is severely restrictive, and is closely related to Beauville's "splitting property' conjecture" [1]. To give an idea: hyperelliptic curves have an MCK decomposition [40, Example 8.16], but the very general curve of genus $\geq 3$ does not have an MCK decomposition [11, Example 2.3]. For more detailed discussion, and examples of varieties with an MCK decomposition, we refer to [40, Section 8], as well as [48], [41], [12], [25], [26], [27], [28], [29], [30], [31], [11], [35].

2.2. Prime Fano threefolds of genus 8. The classification of Fano threefolds is one of the glories of 20th century algebraic geometry [17]. Fano threefolds that are prime (i.e. with Picard group of rank 1 generated by the canonical divisor) come in 10 explicitly described families. In this paper we will be concerned with one of these families:

Theorem 2.5 (Gushel [14], Mukai [32]). Let $Y$ be a smooth projective Fano threefold with $\operatorname{Pic}(Y)=\mathbb{Z}$, of index 1 and genus 8 . Then $Y$ is a dimensionally transverse intersection of the Grassmannian $\operatorname{Gr}(2,6) \subset \mathbb{P}^{14}$ with a codimension 5 linear subspace $\mathbb{P}^{9} \subset \mathbb{P}^{14}$.

Conversely, any smooth dimensionally transverse intersection $\operatorname{Gr}(2,6) \cap \mathbb{P}^{9} \subset \mathbb{P}^{14}$ is a prime Fano threefold of genus 8.

Proof. The last statement is just because $\operatorname{Gr}(2,6)$ is a Fano variety of dimension 8 with Picard number 1 , index 6 and degree 14; the codimension 5 intersection $Y:=\operatorname{Gr}(2,6) \cap \mathbb{P}^{9}$ thus has index 1 and degree $d=14$ (i.e. genus $g=d / 2+1=8$ ). The first statement is proven in [14] and independently in [32]. 
2.3. A birationality. Any prime Fano threefold of genus 8 is birational to a cubic threefold; this was already known to Fano [8], and has been used in many works [15], [16], [38], [21], [23], [24]. The birationality is quite explicit:

Theorem 2.6 (Puts [38], Iskovskikh [16]). Let $Y$ be a prime Fano threefold of genus 8. There exists a smooth cubic threefold $X$ and a birational map

$$
\phi: \quad Y \rightarrow X \text {. }
$$

More precisely, there exist a threefold $Z$ and morphisms

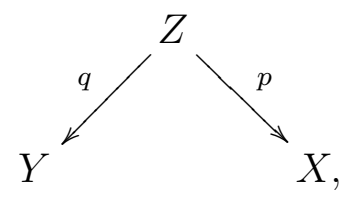

with the following properties:

(i) $p$ is the composition of the blow-up of a quintic elliptic curve $E_{X} \subset X$, and the blow-ups of (strict transforms of) lines $L_{X}^{1}, \ldots, L_{X}^{25} \subset X$; the lines $L_{X}^{j}$ are pairwise disjoint and $E_{X} \cap L_{X}^{j}$ consists of two points for each $j$;

(i) $q$ is the composition of the blow-up of a quintic elliptic curve $E_{Y} \subset Y$, and the blow-ups of (strict transforms of) lines $L_{Y}^{1}, \ldots, L_{Y}^{25} \subset Y$; the lines $L_{Y}^{j}$ are pairwise disjoint and $E_{Y} \cap L_{Y}^{j}$ consists of one point for each $j$;

(iii) $p^{-1}\left(E_{X}\right)=q^{-1}\left(E_{Y}\right)$ and $p^{-1}\left(L_{X}^{j}\right)=q^{-1}\left(L_{Y}^{j}\right), j=1, \ldots, 25$.

Proof. In a nutshell, the argument goes as follows (this can be found in [38], cf. also [16. Chapter III Section 1] and [15, section 3]). Let $V$ be a 6 -dimensional vector space, and let us write $G:=\operatorname{Gr}(2, V) \subset \mathbb{P}\left(\wedge^{2} V\right)$ for the Grassmannian and its Plücker embedding. The projective dual to $G$ is the Pfaffian hypersurface $X_{13} \subset \mathbb{P}\left(\wedge^{2} V^{*}\right)$ (which is a cubic hypersurface of dimension 13, singular along $\left.\operatorname{Gr}\left(2, V^{*}\right) \subset X_{13}\right)$. Given a smooth genus 8 Fano threefold $Y=G \cap h_{1} \cap \cdots \cap h_{5}$, the intersection

$$
X:=X_{13} \cap\left\langle h_{1}^{\vee}, \ldots, h_{5}^{\vee}\right\rangle \quad \subset \mathbb{P}\left(\wedge^{2} V^{*}\right)
$$

is a smooth cubic threefold, called the orthogonal cubic threefold. A point $x \in X$ represents a skew-symmetric form of rank 4 on $V$, whose kernel determines a line $n_{x} \subset \mathbb{P}^{5}$.

The Palatini quartic associated to $Y$ is defined as

$$
W_{4}:=\bigcup_{y \in Y} \ell_{y} \subset \mathbb{P}^{5},
$$

where $\ell_{y} \subset \mathbb{P}(V) \cong \mathbb{P}^{5}$ is the line determined by $y \in Y$. This $W_{4}$ is a quartic hypersurface (singular along a curve), and it turns out that this coincides with

$$
W_{4}=\bigcup_{x \in X} n_{x} \subset \mathbb{P}^{5},
$$

where $X$ is the orthogonal cubic threefold. Choosing a general hyperplane $H \subset \mathbb{P}^{5}$ and setting $W:=W_{4} \cap H$, one can define a map $u_{Y}: Y \rightarrow W$ by sending $y \in Y$ to the intersection point $\ell_{y} \cap H$. Likewise, one can define a map $u_{X}: X \rightarrow W$ by sending $x \in X$ to the intersection point 
$n_{x} \cap H$. One can check (cf. [38]) that the maps $u_{X}$ and $u_{Y}$ are birational, and so one obtains a birationality

$$
Y \stackrel{u_{Y}}{\longrightarrow} W \stackrel{u_{X}}{\longleftarrow} X
$$

It remains to analyze the elimination of indeterminacy of this birational map. The indeterminacy locus of $u_{Y}$ (resp. $u_{X}$ ) is a quintic elliptic curve $E_{Y}$ (resp. $E_{X}$ ). For $H$ sufficiently general, the singular locus of $W$ consists of 25 singular points $w_{1}, \ldots, w_{25}$ (and these points correspond to the $L_{Y}^{j}$ via $u_{Y}$, and to the $L_{X}^{j}$ via $u_{X}$ ). Let $Z \rightarrow W$ be the blow-up of $w_{1}, \ldots, w_{25}$. One can show [38. Section 6] that there are morphisms from $Z$ to the blow-up of $Y$ in $E_{Y}$, and to the blow-up of $X$ in $E_{X}$; this gives the statements (i), (ii), (iii).

\subsection{An isomorphism of motives.}

Proposition 2.7. Let $Y$ be a prime Fano threefold of genus 8, and let $X$ be the orthogonal cubic threefold. There is an isomorphism

$$
h(Y) \stackrel{\cong}{\rightrightarrows} h(X) \text { in } \mathcal{M}_{\text {rat }} \text {. }
$$

Proof. Let $h \in A^{1}(Y)$ denote a hyperplane section (with respect to the embedding $Y \subset \mathbb{P}^{14}$ given by Theorem 2.5). We define

$$
\begin{aligned}
\pi_{Y}^{0} & :=\frac{1}{14} h^{3} \times Y, \\
\pi_{Y}^{2} & :=\frac{1}{14} h^{2} \times h, \\
\pi_{Y}^{4} & :=\frac{1}{14} h \times h^{2}, \\
\pi_{Y}^{6} & :=\frac{1}{14} Y \times h^{3}, \\
\pi_{Y}^{3} & :=\Delta_{Y}-\sum_{j \neq 3} \pi_{Y}^{j} \in A^{3}(Y \times Y),
\end{aligned}
$$

and $h^{j}(Y):=\left(Y, \pi_{Y}^{j}, 0\right) \in \mathcal{M}_{\text {rat }}$. Using a hyperplane section $h \in A^{1}(X)$, we similarly define a decomposition $h(X)=\oplus_{j} h^{j}(X)$.

Let $\iota: D \hookrightarrow Z$ be the inclusion morphism of the exceptional divisor $D:=q^{-1}\left(E_{Y}\right)=$ $p^{-1}\left(E_{X}\right)$ in $Z$ (where the curves $E_{Y}, E_{X}$ are as in Theorem 2.6). The blow-up exact sequence gives equalities

$$
\begin{aligned}
\operatorname{Im}\left(H^{3}(X, \mathbb{Q}) \stackrel{p^{*}}{\rightarrow} H^{3}(Z, \mathbb{Q})\right) & =\operatorname{ker}\left(H^{3}(Z, \mathbb{Q}) \rightarrow H^{3}(D, \mathbb{Q})\right) \\
& =\operatorname{Im}\left(H^{3}(Y, \mathbb{Q}) \stackrel{q^{*}}{\rightarrow} H^{3}(Z, \mathbb{Q})\right),
\end{aligned}
$$

and so in particular there is an isomorphism

$$
p_{*} q^{*}: \quad H^{3}(Y, \mathbb{Q}) \stackrel{\cong}{\rightarrow} H^{3}(X, \mathbb{Q}) .
$$

Using Manin's identity principle, this implies there is an isomorphism of homological motives

$$
\Gamma_{p} \circ{ }^{t} \Gamma_{q}: h^{3}(Y) \stackrel{\cong}{\rightarrow} h^{3}(X) \text { in } \mathcal{M}_{\mathrm{hom}} .
$$


It is known (cf. [45, Theorem 4]) that Fano threefolds have finite-dimensional motives (in the sense of Kimura [20]), and so $h^{3}(X)$ and $h^{3}(Y)$ are finite-dimensional. It follows that there is also an isomorphism of Chow motives

$$
\Gamma_{p} \circ{ }^{t} \Gamma_{q}: h^{3}(Y) \stackrel{\cong}{\rightarrow} h^{3}(X) \text { in } \mathcal{M}_{\text {rat }} .
$$

For $j=0, \ldots, 3$, we have $h^{2 j}(Y) \cong h^{2 j}(X) \cong \mathbb{1}(-j)$. Combined with the above isomorphism $h^{3}(Y) \cong h^{3}(X)$, this proves the proposition.

\subsection{Franchetta property.}

Definition 2.8. Let $\mathcal{X} \rightarrow B$ be a smooth projective morphism, where $\mathcal{X}, B$ are smooth quasiprojective varieties. We say that $\mathcal{X} \rightarrow B$ has the Franchetta property in codimension $j$ if the following holds: for every $\Gamma \in A^{j}(\mathcal{X})$ such that the restriction $\left.\Gamma\right|_{X_{b}}$ is homologically trivial for the very general $b \in B$, the restriction $\left.\Gamma\right|_{b}$ is zero in $A^{j}\left(X_{b}\right)$ for all $b \in B$.

We say that $\mathcal{X} \rightarrow B$ has the Franchetta property if $\mathcal{X} \rightarrow B$ has the Franchetta property in codimension $j$ for all $j$.

This property is studied in [36], [3], [9], [10].

\subsection{Franchetta property for $Y \times Y$.}

Notation 2.9. Let $G$ be the Grassmannian $G:=\operatorname{Gr}(2,6)$, and let $\mathcal{O}_{G}(1)$ be the polarization corresponding to the Plücker embedding $G \subset \mathbb{P}^{14}$. Let

$$
B \subset \bar{B}:=\mathbb{P} H^{0}\left(G, \mathcal{O}_{G}(1)^{\oplus 5}\right)
$$

denote the Zariski open parametrizing smooth dimensionally transverse complete intersections, and let

$$
\mathcal{Y} \rightarrow B
$$

denote the universal family of smooth 3-dimensional complete intersections (in view of Theorem 2.5 this is the universal family of prime Fano threefolds of genus 8).

Proposition 2.10. Let $\mathcal{Y} \rightarrow B$ be the universal family of prime Fano threefolds of genus 8 . The families $\mathcal{Y} \rightarrow B$ and $\mathcal{Y} \times_{B} \mathcal{Y} \rightarrow B$ have the Franchetta property.

Proof. We exploit the relation with cubic threefolds (Theorem 2.6 and Proposition 2.7). For a given $Y_{b}$, let $X_{b}$ denote the orthogonal cubic threefold, let $Z_{b}$ denote the resolution of indeterminacy with morphisms

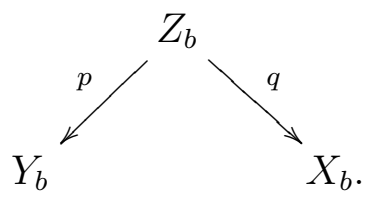

We observe that the construction of Theorem 2.6 makes sense familywise. That is, let us write $\mathcal{X} \rightarrow B$ for the universal family of smooth dimensionally transverse cubic threefolds $X_{b}$ obtained as intersections $X_{b}=X_{13} \cap \mathbb{P}_{b}^{4}$ (here a point $b \in B$ determines a 4-dimensional linear subspace $\mathbb{P}_{b}^{4} \subset \mathbb{P}\left(\wedge^{2} V^{*}\right)$ which is the orthogonal to $\mathbb{P}_{b}^{9} \subset \mathbb{P}\left(\wedge^{2} V\right)$ ). Up to shrinking $B$, we may 
assume the $X_{b}$ avoid the singular locus of $X_{13}$, and thus $\mathcal{X}$ is a smooth quasi-projective variety (it is an open in a projective bundle over $X_{13} \backslash \operatorname{Sing}\left(X_{13}\right)$ ).

Fixing the choice of a hyperplane $H \subset V$, one has a birational map $\mathcal{Y} \rightarrow \mathcal{X}$, and doing the required blow-ups, one obtains a diagram of $B$-schemes

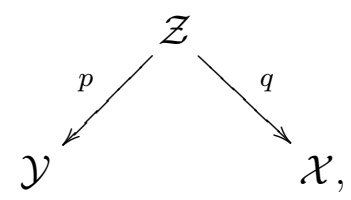

such that the base change along $b \hookrightarrow B$ gives back the diagram (2). In particular, the isomorphism $h(Y) \cong h(X)$ of Proposition 2.7 is generically defined, and so the induced isomorphisms of Chow groups

$$
A^{*}\left(Y_{b}\right) \stackrel{\cong}{\rightarrow} A^{*}\left(X_{b}\right), \quad A^{*}\left(Y_{b} \times Y_{b}\right) \stackrel{\cong}{\rightarrow} A^{*}\left(X_{b} \times X_{b}\right)
$$

send generically defined cycles to generically defined cycles. Consequently, Proposition 2.10 reduces to proving the Franchetta property for the families $\mathcal{X} \rightarrow B$ and $\mathcal{X} \times_{B} \mathcal{X}$. This is the content of the next proposition:

Proposition 2.11. Let $\mathcal{X} \rightarrow B$ be the universal family of orthogonal cubic threefolds, as above. (i) Let $\Gamma \in A^{*}(\mathcal{X})$ be such that the restriction $\left.\Gamma\right|_{X_{b}}$ is homologically trivial, for the very general fiber $X_{b}$. Then

$$
\left.\Gamma\right|_{X_{b}}=0 \text { in } A^{*}\left(X_{b}\right) \text { for all } b \in B .
$$

(ii) Let $\Gamma \in A_{*}\left(\mathcal{X} \times_{B} \mathcal{X}\right) 1$ such that the restriction $\left.\Gamma\right|_{X_{b} \times X_{b}}$ is homologically trivial, for the very general fiber $X_{b}$. Then

$$
\left.\Gamma\right|_{X_{b}}=0 \text { in } A^{*}\left(X_{b} \times X_{b}\right) \text { for all } b \in B .
$$

Proof. (NB: note that this is not the standard presentation of cubic threefolds in $\mathbb{P}^{4}$, and so this does not follow directly from the Franchetta property for the universal cubic in $\mathbb{P}^{4}$ (and its square) !)

(i) Let us assume $j=2$ (since $A^{1}\left(X_{b}\right)=A^{3}\left(X_{b}\right)=\mathbb{Q}$, the statement is non-trivial only for $j=2$ ).

Let $\overline{\mathcal{X}} \rightarrow \bar{B}$ denote the Zariski closure. This is the universal family of all (possibly singular and degenerate) intersections of the Pfaffian $X_{13} \subset \mathbb{P}^{14}$ with a $\mathbb{P}^{4}$, and so $\bar{B} \cong \mathbb{P}^{r}$. Since every point in $X_{13}$ imposes one condition on hyperplanes, the projection $\pi: \overline{\mathcal{X}} \rightarrow X_{13}$ is a $\mathbb{P}^{r-10_{-}}$ bundle.

Now given $\Gamma \in A_{\operatorname{dim} \mathcal{X}_{-2}}(\mathcal{X})$, let $\bar{\Gamma} \in A_{\operatorname{dim} \mathcal{X}_{-2}}(\overline{\mathcal{X}})$ be a cycle restricting to $\Gamma$. The projective bundle formula yields

$$
\bar{\Gamma}=\pi^{*}\left(a_{2}\right)+\pi^{*}\left(a_{1}\right) \cdot \xi \text { in } A_{\operatorname{dim} \mathcal{X}-2}(\overline{\mathcal{X}}),
$$

\footnotetext{
${ }^{1}$ Here we write $A_{*}\left(\mathcal{X} \times_{B} \mathcal{X}\right)$ rather than $A^{*}\left(\mathcal{X} \times_{B} \mathcal{X}\right)$ because $\mathcal{X} \times_{B} \mathcal{X}$ may not be smooth. The Gysin homomorphism $A_{*}\left(\mathcal{X} \times_{B} \mathcal{X}\right) \rightarrow A^{*}\left(X_{b} \times X_{b}\right)$ is still well-defined, because $B$ is smooth.
} 
where $\xi \in A^{1}(\overline{\mathcal{X}})$ is relatively ample with respect to $\pi$ and $a_{j} \in A_{13-j}\left(X_{13}\right)$. Let $h \in A^{1}(\bar{B})$ be a hyperplane section and let $\nu: \overline{\mathcal{X}} \rightarrow \bar{B}$ denote the projection. We have

$$
\nu^{*}(h)=c \xi+\pi^{*}(z) \text { in } \operatorname{Pic}(\overline{\mathcal{X}}),
$$

for some $c \in \mathbb{Q}$ and $z \in A_{12}\left(X_{13}\right)$. It is readily checked that the constant $c$ is non-zero. (Indeed, assume for a moment $c$ were zero. Then we would have $\nu^{*}\left(h^{r}\right)=\pi^{*}\left(z^{r}\right)$ in $H^{2 r}(\overline{\mathcal{X}})$. But the right-hand side is zero, since $r:=\operatorname{dim} \bar{B}>\operatorname{dim} X_{13}=13$, while the left-hand side is non-zero.)

The constant $c$ being non-zero, we can write

$$
\xi=\pi^{*}(z)+\nu^{*}(d) \text { in } A_{\operatorname{dim} \mathcal{X}-1}(\overline{\mathcal{X}}),
$$

where $z \in A_{12}\left(X_{13}\right)$ and $d \in A^{1}(\bar{B})$ are non-zero elements. The restriction of $\nu^{*}(d)$ to a fiber $X_{b}$ is zero, and so we find that

$$
\left.\Gamma\right|_{X_{b}}=\left.a_{2}^{\prime}\right|_{X_{b}} \text { in } A^{2}\left(X_{b}\right),
$$

for some $a_{2}^{\prime} \in A_{11}\left(X_{13}\right)$. (Note that the restriction map $A_{11}\left(X_{13}\right) \rightarrow A^{2}\left(X_{b}\right)$ is well-defined, since $X_{b} \rightarrow X_{13}$ is the base change of $\mathbb{P}^{4} \rightarrow \mathbb{P}^{14}$ which is lci.) The proposition now follows from Lemma 2.12 below.

Lemma 2.12. $A_{11}\left(X_{13}\right) \cong \mathbb{Q}$.

To prove the lemma, we use the resolution of singularities constructed in [22, Section 3]. Let $\widetilde{X}_{13}$ be the variety parametrizing pairs $(\omega, K)$, where $K \subset V$ is a 2-dimensional subspace and $\omega$ is a skew-form containing $K$ in the kernel. The variety $\widetilde{X}_{13}$ is smooth (it is a projective bundle over a Grassmannian). The forgetful morphism $f: \widetilde{X}_{13} \rightarrow X_{13}$ induces a diagram

$$
\begin{array}{ccc}
E & \stackrel{i}{\hookrightarrow} & \widetilde{X}_{13} \\
\left.\downarrow f\right|_{E} & & \downarrow f \\
S:=\operatorname{Sing}\left(X_{13}\right) & \hookrightarrow & X_{13},
\end{array}
$$

where $f$ is an isomorphism over the non-singular locus $X_{13}^{\circ}:=X_{13} \backslash \operatorname{Sing}\left(X_{13}\right)$, and $\left.f\right|_{E}: E \rightarrow$ $S \cong \operatorname{Gr}(2,6)$ is a $\operatorname{Gr}(2,4)$-fibration.

The variety $\widetilde{X}_{13}$, being a projective bundle over a Grassmannian, has trivial Chow groups and so in particular the cycle class map induces an isomorphism

$$
A^{2}\left(\widetilde{X}_{13}\right) \stackrel{\cong}{\rightarrow} H^{4}\left(\widetilde{X}_{13}, \mathbb{Q}\right) \text {. }
$$

The exceptional divisor $E$ has $A_{0}(E) \cong \mathbb{Q}$ (this follows from the fibration result in [46, Theorem 3.2], in view of the fact that both $S \cong \operatorname{Gr}(2,6)$ and the fibers $\operatorname{Gr}(2,4)$ of $\left.f\right|_{E}$ have trivial Chow group of 0 -cycles). The Bloch-Srinivas argument [5] (plus the fact that $h^{p q}(E)=0$ for $p \neq q$ ) then implies that the cycle class maps induce isomorphisms

$$
A^{2}(E) \stackrel{\cong}{\rightarrow} H^{4}(E, \mathbb{Q}) \text {. }
$$


The isomorphisms (3) and (4) fit into a commutative diagram with exact rows

$$
\begin{aligned}
& A_{11}(E) \quad \stackrel{i_{*}}{\rightarrow} \quad A_{11}\left(\tilde{X}_{13}\right) \quad \rightarrow \quad A_{11}\left(X_{13}\right) \quad \rightarrow 0 \\
& \downarrow \cong \quad \downarrow \cong \\
& H_{22}(E, \mathbb{Q}) \stackrel{i_{*}}{\rightarrow} H_{22}\left(\widetilde{X}_{13}, \mathbb{Q}\right) \rightarrow W_{-22} H_{22}\left(X_{13}, \mathbb{Q}\right) \rightarrow 0
\end{aligned}
$$

(Here vertical arrows are cycle class maps, and $W_{*}$ refers to Deligne's weight filtration on homology [37].) It follows that the right vertical arrow is also an isomorphism, and so one is reduced to proving that $W_{-22} H_{22}\left(X_{13}, \mathbb{Q}\right)$ is one-dimensional.

The exact sequence



(where the vertical equalities follow from weak Lefschetz) yields that

$$
H_{22}\left(\widetilde{X}_{13}, \mathbb{Q}\right) \cong H^{4}\left(\widetilde{X}_{13}, \mathbb{Q}\right) \cong H^{4}\left(X_{13}, \mathbb{Q}\right) \oplus H^{2}(S, \mathbb{Q}) \cong \mathbb{Q}^{2}
$$

Since $\left.f\right|_{E}: E \rightarrow S \cong \operatorname{Gr}(2,6)$ is a $\operatorname{Gr}(2,4)$-fibration, we find that

$$
H_{22}(E, \mathbb{Q}) \cong H^{2}(E, \mathbb{Q}) \cong \mathbb{Q}^{2} \text {. }
$$

The arrows labelled $i_{*}$ in (5) cannot be identically zero (indeed, let $h \in A^{1}\left(\widetilde{X}_{13}\right)$ be an ample divisor. Then $i_{*} i^{*}(h)=h \cdot E$ is non-zero in $\left.H_{22}\left(\widetilde{X}_{13}, \mathbb{Q}\right)\right)$, and so we find that $A_{11}\left(X_{13}\right)$ is one-dimensional, proving the lemma.

(ii) We consider again the Zariski closure $B \subset \bar{B} \cong \mathbb{P}^{r}$, and the induced family $\overline{\mathcal{X}} \times_{\bar{B}} \overline{\mathcal{X}} \rightarrow \bar{B}$. Since 2 distinct points in $X_{13}$ impose 2 independent conditions on each hyperplane, the morphism

$$
(\pi, \pi): \quad \overline{\mathcal{X}} \times_{\bar{B}} \overline{\mathcal{X}} \rightarrow X_{13} \times X_{13}
$$

has the structure of a $\mathbb{P}^{r-20}$-bundle over $\left(X_{13} \times X_{13}\right) \backslash \Delta_{X_{13}}$, and a $\mathbb{P}^{r-10}$-bundle over $\Delta_{X_{13}}$.

Using the projective bundle formula, this implies that for any $b \in B$ one has equality

$$
\begin{aligned}
\operatorname{Im}\left(A_{*}\left(\overline{\mathcal{X}} \times_{\bar{B}} \overline{\mathcal{X}} \rightarrow A_{*}\left(X_{b} \times X_{b}\right)\right)=\operatorname{Im}\left(A_{*}\left(X_{13} \times X_{13}\right) \rightarrow A_{*}\left(X_{b} \times X_{b}\right)\right)\right. \\
+\Delta_{*} \operatorname{Im}\left(A_{*}\left(X_{13}\right) \rightarrow A_{*}\left(X_{b}\right)\right),
\end{aligned}
$$

where $\Delta: X_{b} \rightarrow X_{b} \times X_{b}$ denotes the diagonal embedding (the equality (6) is proven exactly as [9. Proposition 4.1]). As we have just seen (in the proof of (i) directly above), there is equality

$$
\operatorname{Im}\left(A_{*}\left(X_{13}\right) \rightarrow A_{*}\left(X_{b}\right)\right)=\langle h\rangle .
$$

Moreover, the fact that $X_{13}$ has a resolution of singularities $\widetilde{X}_{13}$ with trivial Chow groups implies that $X_{13}$ has the Chow-Künneth property:

$$
A_{*}\left(X_{13} \times X_{13}\right)=A_{*}\left(X_{13}\right) \otimes A_{*}\left(X_{13}\right) .
$$


(This is just because $A_{*}\left(\widetilde{X}_{13}\right) \rightarrow A_{*}\left(X_{13}\right)$ is surjective, and $\widetilde{X}_{13}$, being a smooth projective variety with trivial Chow groups, has the Chow-Künneth property.) In view of these two observations, equality (6) reduces to

$$
\operatorname{Im}\left(A_{*}\left(\overline{\mathcal{X}} \times_{\bar{B}} \overline{\mathcal{X}}\right) \rightarrow A_{*}\left(X_{b} \times X_{b}\right)\right)=\left\langle h, \Delta_{X_{b}}\right\rangle .
$$

It now remains to prove that for any smooth cubic threefold $X_{b}$ the $\mathbb{Q}$-subalgebra

$$
R^{*}\left(X_{b} \times X_{b}\right):=\left\langle h, \Delta_{X_{b}}\right\rangle \subset A^{*}\left(X_{b} \times X_{b}\right)
$$

injects into cohomology via the cycle class map. In codimension 3 this is fine, because the diagonal $\Delta_{X_{b}}$ is linearly independent from decomposable cycles $A^{*}\left(X_{b}\right) \otimes A^{*}\left(X_{b}\right)$ in $H^{6}\left(X_{b} \times\right.$ $X_{b}, \mathbb{Q}$ ) (indeed, if the diagonal were decomposable, all of $H^{*}\left(X_{b}, \mathbb{Q}\right)$ would be algebraic, which is absurd since $\left.h^{2,1}\left(X_{b}\right) \neq 0\right)$. For $j \neq 3$, the equality

$$
\Delta_{X_{b}} \cdot\left(p_{j}\right)^{*}(h)=\sum_{i=1}^{4} \frac{1}{3} h^{i} \times h^{4-i} \text { in } A^{4}\left(X_{b} \times X_{b}\right) \quad(j=1,2)
$$

(which follows from the excess intersection formula, cf. [10, Equation (12)]) implies that

$$
R^{j}\left(X_{b} \times X_{b}\right) \subset R^{*}\left(X_{b}\right) \otimes R^{*}\left(X_{b}\right)=\langle h\rangle \otimes\langle h\rangle \quad(j \neq 3),
$$

and hence $R^{j}\left(X_{b} \times X_{b}\right) \rightarrow H^{2 j}\left(X_{b} \times X_{b}, \mathbb{Q}\right)$ is injective also for $j \neq 3$. The proposition is proven.

For later use, we also record the following, which is an easy consequence of the above.

Proposition 2.13. Let $\mathcal{Y} \rightarrow B$ and $\mathcal{X} \rightarrow B$ be as above. The family $\mathcal{Y} \times_{B} \mathcal{X} \rightarrow B$ has the Franchetta property.

Proof. The (generically defined) isomorphism of Proposition 2.7 gives a commutative diagram

$$
\begin{array}{ccc}
A^{*}\left(\mathcal{Y} \times_{B} \mathcal{X}\right) & \rightarrow & A_{*}\left(\mathcal{X} \times_{B} \mathcal{X}\right) \\
\downarrow & & \downarrow \\
A^{*}(Y \times X) & \cong & A^{*}(X \times X) .
\end{array}
$$

The statement thus reduces to the Franchetta property for $\mathcal{X} \times{ }_{B} \mathcal{X}$, which is Proposition 2.11 ,

\subsection{Franchetta property for $Z \times Z$.}

Notation 2.14. Let $\mathcal{Y} \rightarrow B$ be as in Notation 2.9 the universal family of genus 8 prime Fano threefolds. Fixing a hyperplane $H \subset V$, one gets a family $\mathcal{Z} \rightarrow B$ by blowing up $\mathcal{Y}$ along certain closed B-subschemes (cf. proof of Proposition 2.10). (This family $\mathcal{Z} \rightarrow B$ has the property that fiberwise one obtains the resolution of indeterminacy of Proposition 2.6.)

Proposition 2.15. Let $\mathcal{Z} \rightarrow B$ be as in Notation 2.14 The family $\mathcal{Z} \times_{B} \mathcal{Z} \rightarrow B$ has the Franchetta property in codimension $\leq 3$. 
Proof. The $B$-scheme $\mathcal{Z}$ is obtained from the $B$-scheme $\mathcal{Y}$ by blowing up the $B$-subscheme $\mathcal{E} \rightarrow B$ (which is a family of elliptic curves), and then blowing up the strict transforms of the families of lines $\mathcal{L}^{i} \rightarrow B(i=1, \ldots, 25)$. Let us write $Z:=Z_{B}$ for a fiber of $\mathcal{Z} \rightarrow B$ (and likewise $E:=E_{b}, L^{i}:=L_{b}^{i}$ for a fiber of $\mathcal{E} \rightarrow B$ resp. $\mathcal{L} \rightarrow B$ ). The blow-up formula gives an isomorphism of motives (where arrows are the natural ones)

$$
h(Z) \cong h(Y) \oplus h(E)(-1) \oplus \bigoplus_{i=1}^{25} \mathbb{1}(-1) \oplus \bigoplus_{i=1}^{25} \mathbb{1}(-2) \text { in } \mathcal{M}_{\text {rat }}
$$

In particular, for Chow groups of the product we obtain isomorphisms (where arrows are the natural ones)

$$
\begin{aligned}
& A^{j}(Z \times Z) \cong A^{j}(Y \times Y) \oplus A^{j-1}(Y \times E) \oplus A^{j-1}(E \times Y) \oplus A^{j-2}(E \times E) \\
& \oplus \bigoplus A^{*}(Y) \oplus \bigoplus A^{*}(E) \oplus \bigoplus \mathbb{Q} .
\end{aligned}
$$

The isomorphism (7) fits into a commutative diagram

$$
\begin{array}{ccccc}
A^{j}\left(\mathcal{Z} \times{ }_{B} \mathcal{Z}\right) & \stackrel{\phi}{\rightarrow} & A^{j}\left(\mathcal{Y} \times_{B} \mathcal{Y}\right) & \oplus A^{j-1}\left(\mathcal{Y} \times_{B} \mathcal{E}\right) \oplus A^{j-1}\left(\mathcal{E} \times_{B} \mathcal{Y}\right) \oplus A^{j-2}\left(\mathcal{E} \times{ }_{B} \mathcal{E}\right) \oplus \cdots \\
\downarrow & \downarrow & \downarrow & \downarrow \\
A^{j}(Z \times Z) & \stackrel{Ð}{\rightarrow} A^{j}(Y \times Y) & \oplus A^{j-1}(Y \times E) \oplus A^{j-1}(E \times Y) & \oplus & A^{j-2}(E \times E) \oplus \cdots
\end{array}
$$

(Here vertical arrows are fiberwise restrictions, and the arrow $\phi$ is defined because the isomorphism (7) is defined explicitly in terms of the various blow-ups linking $Y$ to $Z$, and these blowups can be performed relatively.) The families $\mathcal{Y} \rightarrow B$ and $\mathcal{Y} \times_{B} \mathcal{Y} \rightarrow B$ have the Franchetta property (Proposition 2.10). Thus, in proving the proposition, we are reduced to proving the following two claims:

Claim 2.16. The families $\mathcal{E} \rightarrow B$ and $\mathcal{E} \times{ }_{B} \mathcal{E} \rightarrow B$ have the Franchetta property in codimension $\leq 1$.

Claim 2.17. The family $\mathcal{Y} \times_{B} \mathcal{E} \rightarrow B$ has the Franchetta property in codimension $\leq 2$.

It remains to prove these two claims. As for Claim 2.16, obviously it suffices to prove the Franchetta property for $\mathcal{E} \times_{B} \mathcal{E} \rightarrow B$. Recall that $E:=E_{Y} \subset Y$ is the indeterminacy locus of the map $u_{Y}: Y \rightarrow W$ described in the proof of Theorem 2.6. We exploit the specific description of $E$ given in [38, Section 6]: letting $H \subset V \cong \mathbb{C}^{6}$ denote the fixed hyperplane defining $W:=W_{4} \cap H$, the elliptic curve $E$ is given as

$$
E=\operatorname{Gr}(2, H) \cap H_{1} \cap \cdots \cap H_{5} \quad \subset \operatorname{Gr}(2, V) \cap H_{1} \cap \cdots \cap H_{5}=: Y .
$$

This implies that $\mathcal{E} \rightarrow B$ coincides with the universal complete intersection of $\operatorname{Gr}(2, H) \subset \mathbb{P}^{14}$ with a codimension 5 linear subspace. As in the proof of Proposition 2.10, the morphism

$$
\overline{\mathcal{E}} \times{ }_{\bar{B}} \overline{\mathcal{E}} \rightarrow \operatorname{Gr}(2, H) \times \operatorname{Gr}(2, H)
$$


has the structure of a $\mathbb{P}^{r-10}$-bundle over $(\operatorname{Gr}(2, H) \times \operatorname{Gr}(2, H)) \backslash \Delta_{\operatorname{Gr}(2, H)}$, and a $\mathbb{P}^{r-5}$-bundle over $\Delta_{\operatorname{Gr}(2, H)}$. Again as in the proof of Proposition 2.10, this implies

$$
\operatorname{Im}\left(A^{*}\left(\overline{\mathcal{E}} \times_{\bar{B}} \overline{\mathcal{E}}\right) \rightarrow A^{*}(E \times E)\right)=\left\langle h, \Delta_{E}\right\rangle=: R^{*}(E \times E) .
$$

It is readily checked that $R^{j}(E \times E)$ injects into cohomology for $j \leq 1$ (it suffices to remark that $\Delta_{E} \in H^{2}(E \times E, \mathbb{Q})$ is not decomposable). This proves Claim 2.16,

As for Claim 2.17, let $g: \operatorname{Gr}(2, H) \hookrightarrow \operatorname{Gr}(2, V)$ denote the inclusion morphism, and let $\Gamma_{g}$ denote its graph. The morphism

$$
\overline{\mathcal{E}} \times_{\bar{B}} \overline{\mathcal{Y}} \rightarrow \operatorname{Gr}(2, H) \times \operatorname{Gr}(2, V)
$$

has the structure of a $\mathbb{P}^{r-10}$-bundle over $(\operatorname{Gr}(2, H) \times \operatorname{Gr}(2, V)) \backslash \Gamma_{g}$, and a $\mathbb{P}^{r-5}$-bundle over $\Gamma_{g}$. This is what is called a stratified projective bundle in [9]. An application of [9, Proposition 5.2] then gives the inclusion

$$
\begin{aligned}
\operatorname{Im}\left(A^{j}\left(\overline{\mathcal{E}} \times_{\bar{B}} \overline{\mathcal{Y}}\right) \rightarrow A^{j}(E \times Y)\right) \subset \operatorname{Im}\left(A^{j}(\operatorname{Gr}(2, H) \times\right. & \left.\operatorname{Gr}(2, V)) \rightarrow A^{j}(E \times Y)\right) \\
& +\operatorname{Im}\left(A^{j-3}\left(\Gamma_{g_{b}}\right) \rightarrow A^{j}(E \times Y)\right)
\end{aligned}
$$

where $g_{b}: E \hookrightarrow Y$ is the inclusion morphism. For $j \leq 2$, the last summand obviously vanishes. Observing that

$$
A^{*}(\operatorname{Gr}(2, H) \times \operatorname{Gr}(2, V))=A^{*}(\operatorname{Gr}(2, H)) \otimes A^{*}(\operatorname{Gr}(2, V)),
$$

and that the Franchetta property holds for $\mathcal{E} \rightarrow B$ and for $\mathcal{Y} \rightarrow B$, this proves Claim 2.17,

\section{MAin RESUlt}

Theorem 3.1. Let $Y$ be a prime Fano threefold of genus 8. Then $Y$ has a multiplicative ChowKünneth decomposition. The induced bigrading on the Chow ring is such that

$$
A_{(0)}^{2}(Y)=\mathbb{Q}\left[c_{2}(Y)\right] \text {. }
$$

Proof. Let us first construct a CK decomposition for $Y$. Letting $h \in A^{1}(Y)$ denote a hyperplane section (with respect to the embedding $Y \subset \mathbb{P}^{14}$ given by Theorem 2.5), we set

$$
\begin{aligned}
\pi_{Y}^{0} & :=\frac{1}{14} h^{3} \times Y, \\
\pi_{Y}^{2} & :=\frac{1}{14} h^{2} \times h, \\
\pi_{Y}^{4} & :=\frac{1}{14} h \times h^{2}, \\
\pi_{Y}^{6} & :=\frac{1}{14} Y \times h^{3}, \\
\pi_{Y}^{3} & :=\Delta_{Y}-\sum_{j \neq 3} \pi_{Y}^{j} \in A^{3}(Y \times Y) .
\end{aligned}
$$


We observe that this CK decomposition is generically defined with respect to the family $\mathcal{Y} \rightarrow B$ (Notation 2.9), i.e. it is obtained by restriction from "universal projectors" $\pi_{\mathcal{Y}}^{j} \in A^{3}\left(\mathcal{Y} \times_{B} \mathcal{Y}\right)$. (This is just because $h$ and $\Delta_{Y}$ are generically defined.)

Writing $h^{j}(Y):=\left(Y, \pi_{Y}^{j}\right) \in \mathcal{M}_{\text {rat }}$, we have

$$
h^{2 j}(Y) \cong \mathbb{1}(-j) \text { in } \mathcal{M}_{\text {rat }}(j=0, \ldots, 3) .
$$

What we need to prove is that this CK decomposition is MCK, i.e.

$$
\pi_{Y}^{k} \circ \Delta_{Y}^{s m} \circ\left(\pi_{Y}^{i} \times \pi_{Y}^{j}\right)=0 \text { in } A^{6}(Y \times Y \times Y) \text { for all } i+j \neq k,
$$

or equivalently that

$$
h^{i}(Y) \otimes h^{j}(Y) \stackrel{\Delta_{Y}^{s m}}{\longrightarrow} h(Y)
$$

coincides with

$$
h^{i}(Y) \otimes h^{j}(Y) \stackrel{\Delta_{Y}^{s m}}{\longrightarrow} h(Y) \rightarrow h^{i+j}(Y) \rightarrow h(Y)
$$

for all $i, j$.

As a first step, let us assume that we have three integers $(i, j, k)$ and at most one of them is equal to 3. The cycle in (9) is generically defined and homologically trivial. The isomorphisms (8) induce an injection

$$
\left(\pi_{Y}^{i} \times \pi_{Y}^{j} \times \pi_{Y}^{k}\right)_{*} A^{6}(Y \times Y \times Y) \hookrightarrow A^{*}(Y),
$$

and sends generically defined cycles to generically defined cycles (this is because the isomorphisms (8) are generically defined). As a consequence, the required vanishing (9) follows from the Franchetta property for $Y$, which is Proposition 2.10(i).

In the second step, let us assume that among the three integers $(i, j, k)$, exactly two are equal to 3 . In this case, using the isomorphisms (8) we find an injection

$$
\left(\pi_{Y}^{i} \times \pi_{Y}^{j} \times \pi_{Y}^{k}\right)_{*} A^{6}(Y \times Y \times Y) \hookrightarrow A^{*}(Y \times Y),
$$

respecting the generically defined cycles. As such, the required vanishing (9) follows from the Franchetta property for $Y \times Y$, which is Proposition 2.10(ii).

(As an aside, we note that in this second step, for most combinations of $(i, j, k)$ the vanishing (9) can also be proven by more elementary means; only for $(i, j, k)=(3,3,4)$ do we need the full force of Proposition 2.10(ii).)

In the third and final step we treat the case $i=j=k=3$. For this case, we exploit the birationality with the cubic threefold $X$, in the guise of the following lemma:

Lemma 3.2. Let $Y$ be a prime Fano threefold of genus 8 , let $X$ be its orthogonal cubic threefold, and let

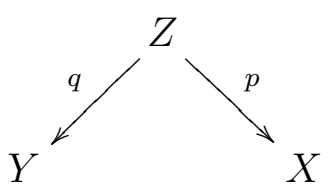

be as in Theorem 2.6. There is equality

$$
{ }^{t} \Gamma_{p} \circ \pi_{X}^{3} \circ \Gamma_{p}={ }^{t} \Gamma_{q} \circ \pi_{Y}^{3} \circ \Gamma_{q} \text { in } A^{3}(Z \times Z)
$$

(where $\pi_{X}^{3}, \pi_{Y}^{3}$ are as above). 
Proof. Both sides of the equality are generically defined (with respect to the family $\mathcal{Z} \rightarrow B$ of Proposition 2.15). The equality holds true in cohomology, because we have seen in equality (1) that

$$
\operatorname{Im}\left(H^{3}(X, \mathbb{Q}) \stackrel{p^{*}}{\rightarrow} H^{3}(Z, \mathbb{Q})\right)=\operatorname{Im}\left(H^{3}(Y, \mathbb{Q}) \stackrel{q^{*}}{\rightarrow} H^{3}(Z, \mathbb{Q})\right) .
$$

The Franchetta property in codimension 3 for $Z \times Z$ (Proposition 2.15) then allows to conclude that the equality of the lemma holds true modulo rational equivalence.

Armed with Lemma 3.2, one can do the final step of the proof. The generically defined CK decomposition $\left\{\pi_{X}^{j}\right\}$ (cf. the proof of Proposition 2.7) is an MCK decomposition (cf. [11], or independently [7]). For brevity, let us write $m:={ }^{t} \Gamma_{p} \circ \pi_{X}^{3} \circ \Gamma_{p} \in A^{3}(Z \times Z)$ for the correspondence of Lemma 3.2. We note that ${ }^{t} \pi_{X}^{3}=\pi_{X}^{3}$ and so ${ }^{t} m=m$. We have equalities

$$
\begin{aligned}
m \circ \Delta_{Z}^{s m} \circ(m \times m) & =(m \times m \times m)_{*}\left(\Delta_{Z}^{s m}\right) \\
& =\left(\left({ }^{t} \Gamma_{p} \circ \pi_{X}^{3} \circ \Gamma_{p}\right)^{\times 3}\right) *\left(\Delta_{Z}^{s m}\right) \\
& =(p, p, p)^{*}\left(\pi_{X}^{3} \times \pi_{X}^{3} \times \pi_{X}^{3}\right)_{*}(p, p, p)_{*}\left(\Delta_{Z}^{s m}\right) \\
& =(p, p, p)^{*}\left(\pi_{X}^{3} \times \pi_{X}^{3} \times \pi_{X}^{3}\right)_{*}\left(\Delta_{X}^{s m}\right) \\
& =(p, p, p)^{*}\left(\pi_{X}^{3} \circ \Delta_{X}^{s m} \circ\left(\pi_{X}^{3} \times \pi_{X}^{3}\right)\right) \\
& =0 \quad \text { in } A^{6}(Z \times Z \times Z) .
\end{aligned}
$$

(Here the first and fifth equality are Lieberman's lemma [47, Lemma 3.3]; the second equality is by definition of $m$; the third follows from general properties of correspondences; the fourth is the fact that $(p, p, p)_{*}\left(\Delta_{Z}^{s m}\right)=\Delta_{X}^{s m}$, and the last equality expresses the fact that $\left\{\pi_{X}^{j}\right\}$ is an MCK decomposition.)

On the other hand, we have equalities

$$
\begin{aligned}
\pi_{Y}^{3} \circ \Delta_{Y}^{s m} \circ\left(\pi_{Y}^{3} \times \pi_{Y}^{3}\right) & =\left(\pi_{Y}^{3} \times \pi_{Y}^{3} \times \pi_{Y}^{3}\right)_{*}\left(\Delta_{Y}^{s m}\right) \\
& =(q, q, q)_{*}(q, q, q)^{*}\left(\left(\pi_{Y}^{3} \times \pi_{Y}^{3} \times \pi_{Y}^{3}\right)_{*}\left(\Delta_{Y}^{s m}\right)\right) \\
& =(q, q, q)_{*}(q, q, q)^{*}\left(\pi_{Y}^{3} \times \pi_{Y}^{3} \times \pi_{Y}^{3}\right)_{*}(q, q, q)_{*}\left(\Delta_{Z}^{s m}\right) \\
& =(q, q, q)_{*}(m, m, m)_{*}\left(\Delta_{Z}^{s m}\right) \\
& =0 \quad \text { in } A^{6}(Y \times Y \times Y) .
\end{aligned}
$$

This proves the required vanishing (9) for $i=j=k=3$, and shows that the CK decomposition is MCK.

Finally, let us prove that $A_{(0)}^{2}(Y)=\mathbb{Q}\left[c_{2}(Y)\right]$. We remark that $c_{2}(Y) \in A_{(0)}^{2}(Y)$ because

$$
\left(\pi_{Y}^{i}\right)_{*} c_{2}(Y)=\left.\left(\pi_{\mathcal{Y}}^{i}\right)_{*} c_{2}\left(T_{\mathcal{Y} / B}\right)\right|_{Y}=0 \text { in } A^{2}(Y) \text { for all } i \neq 4
$$

as follows from the Franchetta property for $\mathcal{Y} \rightarrow B$ (Proposition 2.10). One readily checks that $c_{2}(Y)$ is non-zero (e.g. one can take a smooth anticanonical section $S \subset Y$; if $c_{2}(Y)$ were zero then by adjunction also $c_{2}(S)=0$, which is absurd since $S$ is a K3 surface). Since $A_{(0)}^{2}(Y)$ injects into $H^{4}(Y, \mathbb{Q}) \cong \mathbb{Q}$, it follows that $A_{(0)}^{2}(Y)=\mathbb{Q}\left[c_{2}(Y)\right]$. 


\section{Compatibilities}

The MCK decomposition for $Y$ behaves well with respect to the birationality of Theorem 2.6.

Proposition 4.1. Let $Y$ be a prime Fano threefold of genus 8, let $X$ be the orthogonal cubic threefold and let $\phi: Y \rightarrow X$ be the birational map of Theorem 2.6 One has

$$
\bar{\Gamma}_{\phi} \in A_{(0)}^{3}(Y \times X),
$$

where $Y \times X$ is given the product MCK decomposition.

In particular, one has

$$
\phi_{*} c_{2}(Y) \in \mathbb{Q}\left[c_{2}(X)\right] \subset A^{2}(X) .
$$

Proof. The point is that the map $\phi$, and hence also the cycle $\bar{\Gamma}_{\phi}$, is generically defined (with respect to the base $B$ ). Since the product MCK decomposition is also generically defined, the result (10) follows from the Franchetta property for $Y \times X$ (Proposition 2.13).

For the second part, we have just seen that $A_{(0)}^{2}(Y)=\mathbb{Q}\left[c_{2}(Y)\right]$, and one also has $A_{(0)}^{2}(X)=$ $\mathbb{Q}\left[c_{2}(X)\right]$ (this is just because $c_{2}(X)$ is a sum of intersections of divisors, by adjunction). The result (10) implies that

$$
\phi_{*} A_{(0)}^{2}(Y) \subset A_{(0)}^{2}(X)
$$

(cf. [41, Proposition 1.5]), and so $\phi_{*} c_{2}(Y) \in \mathbb{Q}\left[c_{2}(X)\right]$.

The MCK decomposition for $Y$ also behaves well with respect to birational maps between genus 8 Fano threefolds:

Proposition 4.2. Let $Y_{1}, Y_{2}$ be two prime Fano threefolds of genus 8 such that there is a birational map $\psi: Y_{1} \rightarrow Y_{2}$. There is an isomorphism of motives

$$
\bar{\Gamma}_{\psi}: \quad h^{3}\left(Y_{1}\right) \stackrel{\cong}{\rightarrow} h^{3}\left(Y_{2}\right) \text { in } \mathcal{M}_{\text {rat }},
$$

and one has

$$
\bar{\Gamma}_{\psi} \in A_{(0)}^{3}\left(Y_{1} \times Y_{2}\right)
$$

(where $Y_{1} \times Y_{2}$ is given the product MCK decomposition).

In particular,

$$
\psi_{*} c_{2}\left(Y_{1}\right) \in \mathbb{Q}\left[c_{2}\left(Y_{2}\right)\right] \subset A^{2}\left(Y_{2}\right) .
$$

Proof. Let $X_{1}, X_{2}$ denote the cubic threefold orthogonal to $Y_{1}$ resp. $Y_{2}$, and let $\phi_{i}: Y_{i} \rightarrow X_{i}$ $(i=1,2)$ denote the birational maps of Theorem 2.6. Thanks to the Torelli theorem for cubic threefolds [6], [44] (cf. also [21, Remark 2.19]), $X_{1}$ and $X_{2}$ are isomorphic, and there is a commutative diagram

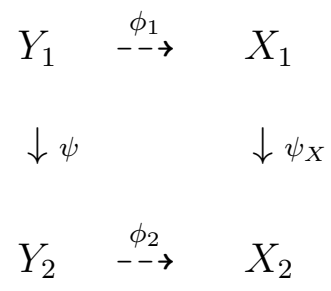


where $\psi_{X}$ is an isomorphism. Since the maps $\phi_{i}$ induce isomorphisms $h^{3}\left(Y_{i}\right) \cong h^{3}\left(X_{i}\right)$ (proof of Proposition 2.7), this proves (11).

Using Manin's identity principle (applied to the diagram obtained from the above diagram by taking the cartesian product with a fixed variety), it follows that there is equality of cycle classes

$$
\bar{\Gamma}_{\psi}={ }^{t} \bar{\Gamma}_{\phi_{2}} \circ \Gamma_{\psi_{X}} \circ \bar{\Gamma}_{\phi_{1}} \text { in } A^{3}\left(Y_{1} \times Y_{2}\right) .
$$

The $\bar{\Gamma}_{\phi_{i}}$ are in $A_{(0)}^{3}\left(Y_{i} \times X_{i}\right)$ (Proposition 4.1), and composition of correspondences preserves the subring $A_{(0)}^{*}()$ [41, Proposition 1.5]. To conclude the truth of equation (12), it only remains to establish the following:

Lemma 4.3. $\Gamma_{\psi_{X}} \in A_{(0)}^{3}\left(X_{1} \times X_{2}\right)$.

To prove the lemma, we observe that the isomorphism $\psi_{X}$ preserves (the canonical divisor and hence) the hyperplane class, which implies that

$$
\pi_{X_{2}}^{j}=\left(\psi_{X} \times \psi_{X}\right)_{*} \pi_{X_{1}}^{j}=\Gamma_{\psi_{X}} \circ \pi_{X_{1}}^{j} \circ{ }^{t} \Gamma_{\psi_{X}} \text { in } A^{3}\left(X_{2} \times X_{2}\right) \quad \forall j
$$

Consequently, we find that

$$
\pi_{X_{2}}^{k} \circ \Gamma_{\psi_{X}} \circ \pi_{X_{1}}^{j}=0 \quad \forall k \neq j
$$

Now writing

$$
\begin{aligned}
\Gamma_{\psi_{X}} & =\left(\sum_{k} \pi_{X_{2}}^{k}\right) \circ \Gamma_{\psi_{X}} \circ\left(\sum_{j} \pi_{X_{1}}^{j}\right) \\
& =\sum_{j} \pi_{X_{2}}^{j} \circ \Gamma_{\psi_{X}} \circ \pi_{X_{1}}^{j} \\
& =\sum_{j}\left(\pi_{X_{1}}^{6-j} \times \pi_{X_{2}}^{j}\right)_{*}\left(\Gamma_{\psi_{X}}\right) \text { in } A^{3}\left(X_{1} \times X_{2}\right),
\end{aligned}
$$

we find that $\Gamma_{\psi_{X}} \in A_{(0)}^{3}\left(X_{1} \times X_{2}\right)$ as requested. This closes the proof of the lemma and the proposition.

Remark 4.4. Let $Y_{1}, Y_{2}$ be 2 genus 8 Fano threefolds that are birational, as in Proposition 4.2 Using the relation with the orthogonal cubic threefold, Kuznetsov proves [21, Corollary 3.18] that there is an equivalence of categories

$$
\operatorname{Kuz}\left(Y_{1}\right) \cong \operatorname{Kuz}\left(Y_{2}\right)
$$

(where $\operatorname{Kuz}\left(Y_{i}\right)$ denotes the Kuznetsov component of the derived category of $Y_{i}$ ). It follows from [4, Theorem 1.1] that the converse is also true: an equivalence of categories $\operatorname{Kuz}\left(Y_{1}\right) \cong \operatorname{Kuz}\left(Y_{2}\right)$ implies that $Y_{1}$ and $Y_{2}$ are birational.

\section{The tAutological Ring}

Corollary 5.1. Let $Y$ be a prime Fano threefold of genus 8 , and let $m \in \mathbb{N}$. Let

$$
R^{*}\left(Y^{m}\right):=\left\langle\left(p_{i}\right)^{*}(h),\left(p_{i j}\right)^{*}\left(\Delta_{Y}\right)\right\rangle \subset A^{*}\left(Y^{m}\right)
$$


be the $\mathbb{Q}$-subalgebra generated by pullbacks of the polarization $h \in A^{1}(Y)$ and pullbacks of the diagonal $\Delta_{Y} \in A^{3}(Y \times Y)$. (Here $p_{i}$ and $p_{i j}$ denote the various projections from $Y^{m}$ to $Y$ resp. to $Y \times Y$ ). The cycle class map induces injections

$$
R^{*}\left(Y^{m}\right) \hookrightarrow H^{*}\left(Y^{m}, \mathbb{Q}\right) \text { for all } m \in \mathbb{N} .
$$

Proof. This is inspired by the analogous result for cubic hypersurfaces [10, Section 2.3], which in turn is inspired by analogous results for hyperelliptic curves [42], [43] (cf. Remark 5.2 below) and for K3 surfaces [49].

As in [10, Section 2.3], let us write $o:=\frac{1}{14} h^{3} \in A^{3}(Y)$, and

$$
\tau:=\Delta_{Y}-\frac{1}{14} \sum_{j=0}^{3} h^{j} \times h^{3-j} \in A^{3}(Y \times Y)
$$

(this cycle $\tau$ is nothing but the projector on the motive $h^{3}(Y)$ considered above). Moreover, let us write

$$
\begin{aligned}
o_{i} & :=\left(p_{i}\right)^{*}(o) \in A^{3}\left(Y^{m}\right), \\
h_{i} & :=\left(p_{i}\right)^{*}(h) \in A^{1}\left(Y^{m}\right), \\
\tau_{i j} & :=\left(p_{i j}\right)^{*}(\tau) \in A^{3}\left(Y^{m}\right) .
\end{aligned}
$$

We define the $\mathbb{Q}$-subalgebra

$$
\bar{R}^{*}\left(Y^{m}\right):=\left\langle o_{i}, h_{i}, \tau_{i j}\right\rangle \quad \subset H^{*}\left(Y^{m}, \mathbb{Q}\right)
$$

(where $i$ ranges over $1 \leq i \leq m$, and $1 \leq i<j \leq m$ ). One can prove (just as [10, Lemma 2.12] and [49, Lemma 2.3]) that the $\mathbb{Q}$-algebra $\bar{R}^{*}\left(Y^{m}\right)$ is isomorphic to the free graded $\mathbb{Q}$-algebra generated by $o_{i}, h_{i}, \tau_{i j}$, modulo the following relations:

$$
\begin{gathered}
o_{i} \cdot o_{i}=0, \quad h_{i} \cdot o_{i}=0, \quad h_{i}^{3}=14 o_{i} ; \\
\tau_{i, j} \cdot o_{i}=0, \quad \tau_{i, j} \cdot h_{i}=0, \quad \tau_{i, j} \cdot \tau_{i, j}=-10 o_{i} \cdot o_{j} ; \\
\tau_{i, j} \cdot \tau_{i, k}=\tau_{j, k} \cdot o_{i} ; \\
\sum_{\sigma \in \mathfrak{S}_{12}} \prod_{i=1}^{6} \tau_{\sigma(2 i-1), \sigma(2 i)}=0 .
\end{gathered}
$$

(NB: with reference to the notation of [10, Lemma 2.12], we have $b_{p r}=\operatorname{dim} H^{3}(Y, \mathbb{Q})=10$.)

To prove Corollary 5.1, it remains to check that all these relations are already verified modulo rational equivalence. The relations (13) take place in $R^{*}(Y)$ and so they follow from the Franchetta property for $Y$ (Proposition 2.10). The relations (14) take place in $R^{*}\left(Y^{2}\right)$. The first and the last relations are trivially verified, because ( $Y$ being Fano) $A^{6}\left(Y^{2}\right)=\mathbb{Q}$. As for the second relation of (14), this follows from the Franchetta property for $Y \times Y$ (Proposition 2.10(ii)). (Alternatively, one can deduce the second relation from the MCK decomposition: the product $\tau \cdot h_{i}$ lies in $A_{(0)}^{4}\left(Y^{2}\right)$, and it is readily checked that $A_{(0)}^{4}\left(Y^{2}\right)$ injects into $H^{8}\left(Y^{2}, \mathbb{Q}\right)$.) 
Relation (15) takes place in $R^{*}\left(Y^{3}\right)$ and follows from the MCK relation. Indeed, we have

$$
\Delta_{Y}^{s m} \circ\left(\pi_{Y}^{3} \times \pi_{Y}^{3}\right)=\pi_{Y}^{6} \circ \Delta_{Y}^{s m} \circ\left(\pi_{Y}^{3} \times \pi_{Y}^{3}\right) \text { in } A^{6}\left(Y^{3}\right),
$$

which (using Lieberman's lemma) translates into

$$
\left(\pi_{Y}^{3} \times \pi_{Y}^{3} \times \Delta_{Y}\right)_{*} \Delta_{Y}^{s m}=\left(\pi_{Y}^{3} \times \pi_{Y}^{3} \times \pi_{Y}^{6}\right)_{*} \Delta_{Y}^{s m} \text { in } A^{6}\left(Y^{3}\right),
$$

which means that

$$
\tau_{13} \cdot \tau_{23}=\tau_{12} \cdot o_{3} \text { in } A^{6}\left(Y^{3}\right) .
$$

Finally, relation (16), which takes place in $R^{*}\left(Y^{12}\right)$, expresses the fact that

$$
\operatorname{Sym}^{12} \operatorname{dim} H^{3}(Y, \mathbb{Q})=0,
$$

where $H^{3}(Y, \mathbb{Q})$ is viewed as a super vector space. To check that this relation is also verified modulo rational equivalence (i.e. relation (16) holds true in $A^{36}\left(Y^{12}\right)$ ), we observe that relation (16) involves a cycle contained in

$$
A^{*}\left(\operatorname{Sym}^{12}\left(h^{3}(Y)\right)\right. \text {. }
$$

But the Chow motive $\left(\operatorname{Sym}^{11} h^{3}(Y)\right.$ and a fortiori) $\operatorname{Sym}^{12} h^{3}(Y)$ is zero because $\operatorname{dim} H^{3}(Y, \mathbb{Q})=$ 10 and $h^{3}(Y)$ is oddly finite-dimensional (all Fano threefolds have finite-dimensional motive [45, Theorem 4]). This ends the proof.

Remark 5.2. Given a curve $C$ and an integer $m \in \mathbb{N}$, one can define the tautological ring

$$
R^{*}\left(C^{m}\right):=\left\langle\left(p_{i}\right)^{*}\left(K_{C}\right),\left(p_{i j}\right)^{*}\left(\Delta_{C}\right)\right\rangle \quad \subset A^{*}\left(C^{m}\right)
$$

(where $p_{i}, p_{i j}$ denote the various projections from $C^{m}$ to $C$ resp. $C \times C$ ). Tavakol has proven [43, Corollary 6.4] that if $C$ is a hyperelliptic curve, the cycle class map induces injections

$$
R^{*}\left(C^{m}\right) \hookrightarrow H^{*}\left(C^{m}, \mathbb{Q}\right) \text { for all } m \in \mathbb{N} .
$$

On the other hand, there are many (non hyperelliptic) curves for which the tautological ring $R^{*}\left(C^{3}\right)$ does not inject into cohomology (this is related to the non-vanishing of the Ceresa cycle, cf. [43, Remark 4.2] and also [11, Example 2.3 and Remark 2.4]).

Corollary 5.1 shows that genus 8 Fano threefolds behave similarly to hyperelliptic curves. It would be interesting to understand what happens for other Fano threefolds: is Corollary 5.1 true for all of them or not? (This is related to Question 1.1.)

\section{QUESTION}

Question 6.1. Let $Y$ be a prime Fano threefold of genus 8, and let $S \subset Y$ be a smooth anticanonical divisor. Then $S$ is a K3 surface (and the general genus $8 \mathrm{~K} 3$ surface arises in this way). Is it true that

$$
\operatorname{Im}\left(A^{1}(S) \rightarrow A^{2}(Y)\right)=\mathbb{Q}\left[h^{2}\right] ? ?
$$

(To prove this, it would suffice to prove that the graph of the inclusion morphism $S \hookrightarrow Y$ is in $A_{(0)}^{3}(S \times Y)$, with respect to the product MCK decomposition. I have not been able to do this.)

This question also makes sense for cubic threefolds and their anticanonical divisors. 
Acknowledgements. Thanks to Charles Vial and the referee for helpful comments. This note is dedicated to Y.: you know why...(-)

\section{REFERENCES}

[1] A. Beauville, On the splitting of the Bloch-Beilinson filtration, in: Algebraic cycles and motives (J. Nagel and C. Peters, editors), London Math. Soc. Lecture Notes 344, Cambridge University Press 2007,

[2] A. Beauville and C. Voisin, On the Chow ring of a K3 surface, J. Alg. Geom. 13 (2004), 417—426,

[3] N. Bergeron and Z. Li, Tautological classes on moduli space of hyperkähler manifolds, Duke Math. J., arXiv:1703.04733,

[4] M. Bernardara, E. Macrì, S. Mehrotra and P. Stellari, A categorical invariant for cubic threefolds, Adv. Math. 229 no 2 (2012), 770-803,

[5] S. Bloch and V. Srinivas, Remarks on correspondences and algebraic cycles, American Journal of Mathematics Vol. 105, No 5 (1983), 1235-1253,

[6] H. Clemens and P. Griffiths, The intermediate Jacobian of the cubic threefold, Annals of Math. 95 (1972), $281-356$,

[7] H. Diaz, The Chow ring of a cubic hypersurface, International Math. Research Notices,

[8] G. Fano, Sulle sezione spaziale della varietà grassmanniana delle rette dello spazio a cinque dimensioni, Rend. Acc. Naz. Lincei 11 (1930), 329-335,

[9] L. Fu, R. Laterveer and Ch. Vial, The generalized Franchetta conjecture for some hyper-Kähler varieties (with an appendix joint with M. Shen), Journal Math. Pures et Appliquées (9) 130 (2019), 1-35,

[10] L. Fu, R. Laterveer and $\mathrm{Ch}$. Vial, The generalized Franchetta conjecture for some hyper-Kähler varieties, II, Journal de l'Ecole Polytechnique-Mathématiques 8 (2021), 1065-1097,

[11] $\mathrm{L}$. Fu, R. Laterveer and $\mathrm{Ch}$. Vial, Multiplicative Chow-Künneth decompositions and varieties of cohomological K3 type, Annali Mat. Pura ed Applicata, doi: 10.1007/s10231-021-01070-0,

[12] L. Fu, Z. Tian and Ch. Vial, Motivic hyperkähler resolution conjecture for generalized Kummer varieties, arXiv:1608.04968,

[13] W. Fulton, Intersection theory, Springer-Verlag Ergebnisse der Mathematik, Berlin Heidelberg New York Tokyo 1984,

[14] N. Gushel, On Fano threefolds of genus 8, St. Petersburg Math. J. 4 no 1 (1993), 115-129,

[15] A. Iliev and L. Manivel, Pfaffian lines and vector bundles on Fano threefolds of genus 8, J. Alg. Geom. 16 (2007), 499-530,

[16] V. Iskovskikh, Birational automorphisms of three-dimensional algebraic varieties, J. Sov. Math. 13 (1980), 815-868,

[17] V. Iskovskih and Yu. Prokhorov, Algebraic Geometry V: Fano varieties, Encyclopaedia of Math. Sciences 47, Springer-Verlag, Berlin 1999,

[18] U. Jannsen, Motivic sheaves and filtrations on Chow groups, in: Motives (U. Jannsen et alii, eds.), Proceedings of Symposia in Pure Mathematics Vol. 55 (1994), Part 1,

[19] U. Jannsen, On finite-dimensional motives and Murre's conjecture, in: Algebraic cycles and motives (J. Nagel and C. Peters, editors), Cambridge University Press, Cambridge 2007,

[20] S.-I. Kimura, Chow groups are finite dimensional, in some sense, Math. Ann. 331 no 1 (2005), 173-201,

[21] A. Kuznetsov, Derived categories of cubic and $V_{14}$ threefolds, Proc. V.A. Steklov Inst. Math 246 (2004), $183-207$,

[22] A. Kuznetsov, Homological projective duality for Grassmannians of lines, arXiv:0610957,

[23] A. Kuznetsov, Derived categories of Fano threefolds, Proc. V. A. Steklov Inst. Math 264 (2009), 110_ 122 ,

[24] A. Kuznetsov, Y. Prokhorov and C. Shramov, Hilbert schemes of lines and conics and automorphism groups of Fano threefolds, Japanese Journal of Mathematics 13 no 1 (2018), 109—185, 
[25] R. Laterveer, A remark on the Chow ring of Küchle fourfolds of type $d 3$, Bulletin Australian Math. Soc. 100 no. 3 (2019), 410-418,

[26] R. Laterveer and Ch. Vial, On the Chow ring of Cynk-Hulek Calabi-Yau varieties and Schreieder varieties, Canadian Journal of Math. 72 no 2 (2020), 505-536,

[27] R. Laterveer, Algebraic cycles and Verra fourfolds, Tohoku Math. J. 72 no. 3 (2020), 451—485,

[28] R. Laterveer, On the Chow ring of certain Fano fourfolds, Ann. Univ. Paedagog. Crac. Stud. Math. 19 (2020), 39-52,

[29] R. Laterveer, Algebraic cycles and Gushel-Mukai fivefolds, Journal of Pure and Applied Algebra 225 no. 5 (2021), doi: 10.1016/j.jpaa.2020.106582,

[30] R. Laterveer, Algebraic cycles and intersections of 2 quadrics, Mediterranean Journal of Mathematics (2021), doi: 10.1007/s00009-021-01787-5,

[31] R. Laterveer, Algebraic cycles and intersections of a quadric and a cubic, Forum Mathematicum 33 no. 3 (2021), 845-855,

[32] S. Mukai, New developments in the theory of Fano threefolds: vector bundle method and moduli problems, Sugaku Expositions 15 no 2 (2002), 125-150,

[33] J. Murre, On a conjectural filtration on the Chow groups of an algebraic variety, parts I and II, Indag. Math. 4 (1993), 177-201,

[34] J. Murre, J. Nagel and C. Peters, Lectures on the theory of pure motives, Amer. Math. Soc. University Lecture Series 61, Providence 2013,

[35] A. Negut, G. Oberdieck and Q. Yin, Motivic decompositions for the Hilbert scheme of points of a K3 surface, arXiv:1912.09320v1,

[36] N. Pavic, J. Shen and Q. Yin, On O'Grady's generalized Franchetta conjecture, Int. Math. Res. Notices (2016), 1-13,

[37] C. Peters and J. Steenbrink, Mixed Hodge Theory, Ergebnisse der Math. 52, Springer Verlag 2008,

[38] P. Puts, On some Fano-threefolds that are sections of Grassmannians, Indag. Math. 44 (1982), 77-90,

[39] T. Scholl, Classical motives, in: Motives (U. Jannsen et alii, eds.), Proceedings of Symposia in Pure Mathematics Vol. 55 (1994), Part 1,

[40] M. Shen and Ch. Vial, The Fourier transform for certain hyperKähler fourfolds, Memoirs of the AMS 240 (2016), no.1139,

[41] M. Shen and Ch. Vial, The motive of the Hilbert cube $X^{[3]}$, Forum Math. Sigma 4 (2016), 55 pp.,

[42] M. Tavakol, The tautological ring of the moduli space $M_{2}^{r t}$, International Math. Research Notices 178 (2013),

[43] M. Tavakol, Tautological classes on the moduli space of hyperelliptic curves with rational tails, J. Pure Applied Algebra 222 no 8 (2018), 2040-2062,

[44] A. Tyurin, Geometry of the Fano surface of a nonsingular cubic $F \subset \mathbb{P}^{4}$ and Torelli theorems for Fano surfaces and cubics, Izv. USSR Math. 35 (1971), 498-529,

[45] Ch. Vial, Projectors on the intermediate algebraic Jacobians, New York J. Math. 19 (2013), 793—822,

[46] Ch. Vial, Algebraic cycles and fibrations, Documenta Math. 18 (2013), 1521-1553,

[47] Ch. Vial, Remarks on motives of abelian type, Tohoku Math. J. 69 (2017), 195-220,

[48] Ch. Vial, On the motive of some hyperkähler varieties, J. Reine Angew. Math. 725 (2017), 235-247,

[49] Q. Yin, Finite-dimensionality and cycles on powers of K3 surfaces, Comment. Math. Helv. 90 (2015), 503-511.

Institut de Recherche Mathématique Avancée, CNRS - Université de Strasbourg, 7 Rue RENÉ DESCARTES, 67084 STRASBOURG CEDEX, FRANCE.

Email address: robert. laterveer@math.unistra.fr 